\title{
Activation of Mst11 and Feedback Inhibition of Germ Tube Growth in Magnaporthe oryzae
}

\author{
Linlu Qi, ${ }^{1,2}$ Yangseon Kim, ${ }^{2}$ Cong Jiang, ${ }^{2,3}$ Yang Li, ${ }^{2}$ Youliang Peng, ${ }^{1}$ and Jin-Rong $\mathrm{Xu}^{2,3}$ \\ ${ }^{1}$ MOA Key Laboratory of Plant Pathology, China Agricultural University, Beijing 100193, China; ${ }^{2}$ Department of Botany and \\ Plant Pathology, Purdue University, West Lafayette, IN 47907, U.S.A.; ${ }^{3}$ NWAFU-PU Joint Research Center, Northwestern A\&F \\ University, Yangling, Shaanxi 712100, China
}

Submitted 12 March 2015. Accepted 24 March 2015.

\begin{abstract}
Appressorium formation and invasive growth are two important steps in the infection cycle of Magnaporthe oryzae that are regulated by the Mst11-Mst7-Pmk1 mitogen-activated protein kinase (MAPK) pathway. However, the molecular mechanism involved in the activation of Mst11 MAPK kinase kinase is not clear in the rice blast fungus. In this study, we functionally characterized the regulatory region of Mst11 and its selfinhibitory binding. Deletion of the middle region of Mst11, which contains the Ras-association (RA) domain and two conserved phosphorylation sites ( $\mathbf{S 4 5 3}$ and $\mathrm{S458}$ ), blocked Pmk1 activation and appressorium formation. However, the $M_{S T 11^{\Delta \mathrm{RA}}}$ transformant MRD-2 still formed appressoria, although it was reduced in virulence. Interestingly, over $50 \%$ of its germ tubes branched and formed two appressoria by $48 \mathrm{~h}$, which was suppressed by treatments with exogenous cAMP. The G18V dominant active mutation enhanced the interaction of Ras2 with Mst11, suggesting that Mst11 has stronger interactions with the activated Ras2. Furthermore, deletion and site-directed mutagenesis analyses indicated that phosphorylation at $\mathbf{S 4 5 3}$ and $\mathbf{S 4 5 8}$ of Mst11 is important for appressorium formation and required for the activation of Pmk1. We also showed that the N-terminal region of Mst11 directly interacted with its kinase domain, and the $\mathbf{S 7 8 9 G}$ mutation reduced their interactions. Expression of the MST11 ${ }^{\mathrm{S789G}}$ allele rescued the defect of the mst11 mutant in plant infection and resulted in the formation of appressoria on hydrophilic surfaces, suggesting the gain-of-function effect of the S789G mutation. Overall, our results indicate that the interaction of Mst11 with activated Ras2 and phosphorylation of $S 453$ and $S 458$ play regulatory roles in Mst11 activation and infection-related morphogenesis, possibly by relieving its self-inhibitory interaction between its $\mathrm{N}$-terminal region and the $\mathrm{C}$-terminal kinase domain. In addition, binding of Mst11 to Ras2 may be involved in the feedback inhibition of cAMP signaling and further differentiation of germ tubes after appressorium formation.
\end{abstract}

Rice blast is caused by the fungal pathogen Magnaporthe oryzae. It occurs worldwide and is one of the most severe fungal diseases of rice. The rice-rice blast pathosystem has been

Linlu Qi and Yangseon Kim contributed equally to this work.

Corresponding author: J.-R. Xu; Telephone: +1.765.496.6918; E-mail: jinrong@purdue.edu

*The $\boldsymbol{e}$-Xtra logo stands for "electronic extra" and indicates that three supplementary figures are published online.

(c) 2015 The American Phytopathological Society developed as a model to study fungal-plant interactions (Dean et al. 2005; Ebbole 2007; Valent and Khang 2010). In the infection cycle of $M$. oryzae, appressorium formation and invasive growth are two critical infection stages. Germ tubes attached to rice leaves can recognize surface hydrophobicity and differentiate into specialized infection structures called appressoria. The fungus generates enormous turgor pressure in melanized appressoria by utilization of the carbohydrate storage in conidia to accumulate a high concentration of glycerol for physical penetration of the cuticle and plant cell wall (Wilson and Talbot 2009). After penetration, M. oryzae colonizes plant cells as a hemibiotrophic pathogen with invasive hyphae that are enveloped in the extra-invasive hyphal membrane produced by the host (Kankanala et al. 2007). The pathogens secreted various effectors to suppress plant defense responses (Yi and Valent 2013; Zhang and Xu 2014), and eventually, extensive growth of invasive hyphae leads to lesion formation.

In the past, several signal transduction pathways regulating different steps of infection-related morphogenesis, including surface recognition, appressorium formation, appressorial penetration, and invasive growth, have been characterized in M. oryzae (Li et al. 2012; Wilson and Talbot 2009). Similar to other plant-pathogenic fungi (Rispail et al. 2009), both the cAMP-PKA (protein kinase A) pathway and PMK1 mitogenactivated protein kinase (MAPK) cascade play important roles in surface recognition, appressorium development, and invasive growth in M. oryzae (Choi and Dean, 1997; Franck et al. 2013; Thines et al. 2000). Treatment with exogenous cAMP stimulates appressorium formation on hydrophilic surfaces and several key components of the cAMP-PKA pathway have been functionally characterized for their roles in surface recognition and the initiation of appressorium formation (Mitchell and Dean, 1995; Xu et al. 1997). The Mst11-Mst7-Pmk1 MAPK cascade regulates the late stages of appressorium formation, appressorial penetration, and invasive growth (Liu et al. 2011; Zhao and Xu 2007). Mutants disrupted in the $P M K 1$ pathway fail to form appressoria and grow invasively in rice plants, but they still respond to exogenous cAMP for germ-tube tip deformation and formation of apical swollen bodies. Studies in other ascomycetous plant-pathogenic fungi, including Botrytis cinerea, Claviceps purpurea, Stagonospora nodorum, and Fusarium species, have shown that the PMK1 pathway is well conserved for regulating various plant infection processes (Jenczmionka et al. 2003; Mey et al. 2002; Solomon et al. 2005; Zheng et al. 2000).

In $M$. oryzae, Pmk1 is activated by the upstream MAPK kinase (MEK) Mst7 and MEK kinase Mst11 (Zhao et al. 2005). Both Mst7 and Mst11 strongly interact with the adaptor protein 
Mst50, which is also essential for the activation of Pmk1 and plant infection (Park et al. 2006). The interaction of Mst7 with Pmk1 is mediated by an N-terminal MAPK docking site (Zhao and $\mathrm{Xu}$ 2007), and whether Mst50 plays a role in stabilizing the Mst7-Pmk1 interaction during appressorium formation is not clear. Nevertheless, neither of the two p21-activated kinase (PAK) genes, CHMI and MST20, is essential for appressorium formation ( $\mathrm{Li}$ et al. 2004). In fact, the mst20 mutant had no obvious defects in growth and plant infection, but the chml mutant was defective in conidium morphology, appressorium morphogenesis, and pathogenesis. Therefore, the activation of the Mst11 MEK kinase is not clear in M. oryzae. MST11 interacts with the two RAS genes, RAS1 and RAS2 (Zhao et al. 2005). Whereas rasl deletion mutants have no detectable phenotype, deletion of RAS2 is lethal (Zhou et al. 2014). Expression of a dominant active $R A S 2$ leads to improper activation of Pmk1 and appressorium formation on hydrophilic surfaces and aerial hyphae in the wild type but not in the $p m k 1$ or $c p k A$ mutant. Dominant active mutations in RAS2 may enable $M$. oryzae to bypass the requirement of surface hydrophobicity and attachment for appressorium formation (Zhou et al. 2014).

Both MST11 and MST50 contain a sterile alpha motif (SAM) and a Ras-association (RA) domain. The mstl1 and mst50 mutants have similar defects in appressorium formation and pathogenesis (Park et al. 2006). Unlike the mst11 or mst50 mutants, which are blocked in appressorium formation, the mst11/MST11 $1^{\Delta \mathrm{RA}}$ transformant still forms appressoria and infects rice seedlings (Zhao et al. 2005). In Saccharomyces cerevisiae, phosphorylation of Ste11 (ortholog of Mst11) by the Ste20 PAK is known to release the self-inhibitory binding between the kinase domain and regulatory region (van Drogen et al. 2000). In Schizosaccharomyces pombe, both Ste4 (ortholog of Mst50) and Ras1 can activate the Byr2 MEK kinase (Gronwald et al. 2001; Ramachander et al. 2002). Byr2, a Ste11 ortholog, functions upstream from the Spk1 pheromone-response MAPK pathway. It directly interacts with Ste 4 and Ras1 and point mutations in the RA domain of Byr2 abolish its interaction with Ras1. In the corn smut fungus Ustilago maydis, the K481E mutation in the RA domain of Kpp4 (ortholog of Ste11) reduces the virulence and formation of dikaryotic hyphae and conjugation tubes (Andrews et al. 2000; Müller et al. 2003). These observations indicate that the RA domain of the MEK kinase plays a role in this important MAPK pathway.

Table 1. Magnaporthe oryzae strains used in this study

\begin{tabular}{|c|c|c|}
\hline Strain & Genotype & Reference \\
\hline $70-15$ & Wild type, MAT1-1 & $\begin{array}{l}\text { Chao and Ellingboe } \\
1991\end{array}$ \\
\hline yk86 & mst11 deletion mutant of $70-15$ & Zhao et al. 2005 \\
\hline MF103 & mst50 deletion mutant of $70-15$ & Park et al. 2006 \\
\hline Yk86C & $\begin{array}{l}\text { mst11/MST11 complemented } \\
\text { transformant }\end{array}$ & Zhao et al. 2005 \\
\hline RP37 & $\begin{array}{l}\text { MST1 } 1^{\triangle \mathrm{MR}(263-565)} \text { transformant of } \\
\text { yk86 }\end{array}$ & This study \\
\hline PH29 & $\begin{array}{l}\text { MST11 } \\
\text { yk86 }\end{array}$ & This study \\
\hline PD65 & $\begin{array}{l}\text { MST11 } 1^{\text {S453D S458D }} \text { transformant of } \\
\text { yk86 }\end{array}$ & This study \\
\hline MRD-2 & $\begin{array}{l}\text { MST11 } \\
\text { yk86 (263-353) }\end{array}$ & Zhao et al. 2005 \\
\hline MRD-5 & $\begin{array}{l}\text { MST11 } \\
\text { yk86 }\end{array}$ & Zhao et al. 2005 \\
\hline NT48 & MST11 $1^{\Delta 6-583}$ transformant of yk86 & This study \\
\hline WY54 & $M S T 11^{\mathrm{S} 789 \mathrm{G}}$ transformant of $70-15$ & This study \\
\hline CA11 & MST1 $^{\mathrm{S} 789 \mathrm{G}}$ transformant of $\mathrm{yk} 86$ & This study \\
\hline MC04 & $\begin{array}{l}\text { MST11 } 1^{\mathrm{S} 789 \mathrm{G}} \text { in the } m s t 50 \text { mutant } \\
\text { MF103 }\end{array}$ & This study \\
\hline
\end{tabular}

This study seeks better understanding of the activation of the Mst11 MEK kinase in M. oryzae. Deletion of the entire middle region but not the RA domain blocked the activation of Pmk1 and appressorium formation, suggesting its importance in Mst11 function. Interestingly, the $M S T 11^{\Delta \mathrm{RA}}$ transformant MRD-2 was defective in arresting germ-tube growth and formed multiple appressoria on branched germ tubes. Deletion and point mutation analyses showed that two conserved phosphorylation sites are required for the activation of Mst11 and invasive growth. We also showed enhanced interaction of Mst11 with Ras2 ${ }^{\mathrm{G} 18 \mathrm{~V}}$ and the self-inhibitory binding between the $\mathrm{N}$-terminal region of Mst11 and its kinase domain. The S789G mutation next to the Asp-Phe-Gly (DFG) motif (residues 790 to 792, a conserved kinase-inhibitor binding site) diminished the self-interaction of Mst11 in yeast two-hybrid assays. It also resulted in the formation of appressoria on hydrophilic surfaces in vivo. These results indicate that phosphorylation in the middle region of Mst11 by one or more unknown protein kinases and its association with Ras2 is important for the activation of Mst11 and regulation of appressorium formation and invasive growth in $M$. oryzae. Binding of Mst11 to Ras2 may also play a role in the feedback inhibition of germ-tube branching or formation of multiple appressoria.

\section{RESULTS}

\section{The RA domain of Mst11 is important}

for suppressing germ-tube branching.

The $m s t 11 / M S T 11^{\Delta \mathrm{RA}}$ transformant MRD-2 (Table 1) generated in a previous study (Zhao et al. 2005) still formed appressoria. However, we noticed that germ tubes of strain MRD-2 often branched and formed two appressoria on hydrophobic plastic coverslips (Fig. 1A). After the formation of an appressorium at the tip, approximately $70 \%$ of the germ tubes produced a branch by $24 \mathrm{~h}$ in strain MRD-2 (Fig. 1A; Table 2). By 48 h, over $50 \%$ of these branched germ tubes formed two appressoria (Fig. 1A). Under the same conditions, germ tubes of wild-type strain 70-15 normally formed a single terminal appressorium at the tip (Fig. 1A). Less than $2.3 \% \pm 0.5 \%$ of the $70-15$ germ tubes branched or formed two appressoria on a branched germ tube (Table 2).

We also assayed appressorium formation on onion epidermal cells with the MRD-2 mutant. By 48 h, over $70 \%$ of the MRD-2 germ tubes branched and approximately $58 \%$ of them produced two appressoria on branched germ tubes (Fig. 1B; Table 2), suggesting that RA deletion had the same effect on plant or artificial surfaces. The mst 11/MST1 $1^{\Delta \mathrm{RA}}$ transformant MRD-2 also was defective in plant penetration. On onion epidermal cells, $68.7 \% \pm 8.7 \%$ of appressoria penetrated and formed infectious hyphae in underlying onion epidermal cells by $48 \mathrm{~h}$. Under the same conditions, only $30.5 \% \pm 3.2 \%$ of the appressoria produced by the mst 11/MST1 $1^{\Delta \mathrm{RA}}$ transformant MRD-2 developed invasive hyphae inside onion epidermal cells (Table 2). When assayed by incipient cytorrhysis, strain MRD-2 was reduced in appressorium turgor pressure (Supplementary Fig. S1). Therefore, although it is dispensable for appressorium formation, the RA domain of Mst11 plays a role in preventing germ tubes from branching or further growth after appressorium formation at the tip, which is likely important for the generation of appressorium turgor pressure required for penetration.

\section{The mst11/MST11 ${ }^{\mathrm{ARA}}$ transformant MRD-2}

\section{is reduced in virulence.}

To determine whether deletion of the RA domain of MST11 will affect virulence, 2-week-old seedlings of rice cultivar Nipponbare were used for spray-inoculation assays. At 7 days postinoculation (dpi), typical blast lesions were observed on leaves sprayed with the wild-type strain 70-15 and 
mst11-complemented transformant Yk86C (Fig. 2A). Under the same conditions, the mst 11 deletion mutant was nonpathogenic and strain MRD-2 was reduced in virulence (Fig. 2A). When quantified, the number of lesions caused by $70-15$ and strain MRD-2 were $47.5 \pm 3.2$ and $31.5 \pm 3.0$ per 5 -cm leaf tip, respectively (Fig. 2B; Table 2). These data suggest that the $M S T 11^{\triangle \mathrm{RA}}$ allele MRD-2 only partially complemented the defects of the mst11 mutant in plant infection. Therefore, consistent with its effects on appressorium formation and penetration, deletion of the RA domain of MST11 resulted in reduced virulence.

\section{Exogenous cAMP treatment reduces germ-tube branching in MRD-2.}

In $M$. oryzae, cAMP signaling is known to regulate surface recognition and the initiation of appressorium formation (Lee and Dean 1993). To test the effect of exogenous cAMP on

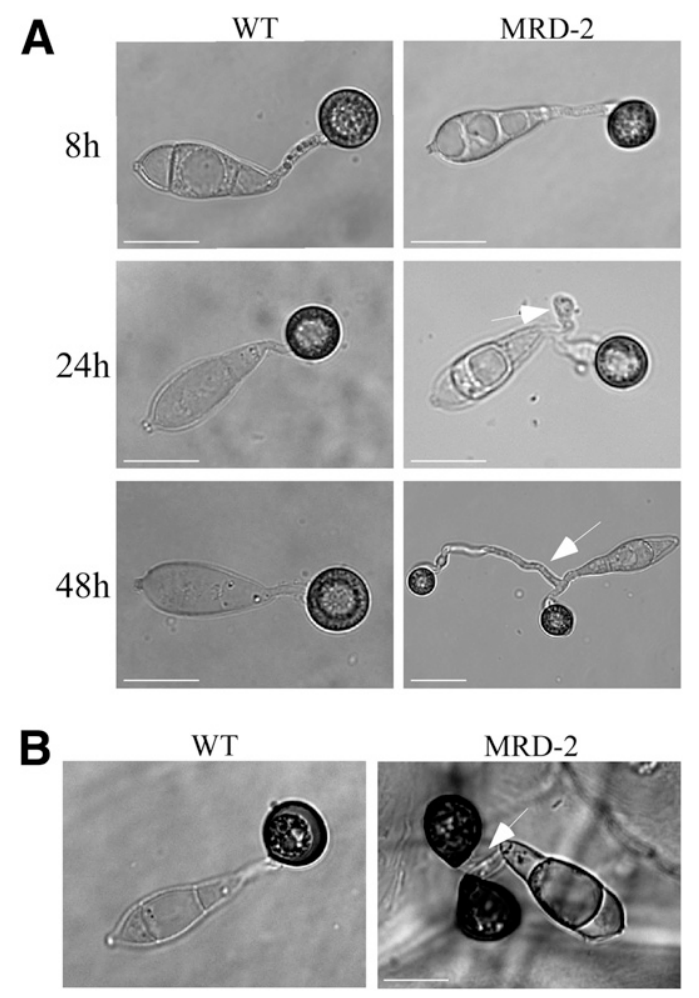

Fig. 1. Appressorium formation assays with the $m s t 11 / M S T 11^{\Delta \mathrm{RA}}$ transformant MRD-2. A, Conidia of the wild-type stain 70-15 and transformant MRD-2 were incubated on hydrophobic plastic coverslips and were assayed for appressorium formation at the indicated times. Branched germ tubes are marked with arrowheads. B, Appressorium formation assays on onion epidermal cells. After incubation for $36 \mathrm{~h}$, over $50 \%$ of branched germ tubes formed two appressoria in MRD-2. Bar $=10 \mu \mathrm{m}$ in all the panels. germ-tube branching, we assayed appressorium formation with 70-15 and strain MRD-2 on the hydrophobic side of GelBond membranes. Without cAMP treatment, approximately $50 \%$ of conidia produced branched germ tubes in strain MRD-2. In the presence of $5 \mathrm{mM}$ cAMP, only $5.7 \% \pm 0.9 \%$ of MRD- 2 germ tubes branched and formed two appressoria per germ tube (Fig. 3 ). In wild-type strain 70-15, approximately 2.0 and $1.7 \%$ of the germ tubes branched with or without $5 \mathrm{mM}$ cAMP (Fig. 3). These data indicated that exogenous cAMP could suppress germ-tube branching and formation of secondary appressoria in the $m$ st 11/MST1 $1^{\triangle \mathrm{RA}}$ transformant MRD-2.

\section{The G18V mutation of Ras2 enhances its interaction with Mst11.}

In M. oryzae, Mst11 interacts with Ras2 in yeast two-hybrid assays and expression of a dominant active Ras 2 stimulates appressorium formation, indicating that Ras2 functions upstream from both the cAMP signaling and Pmk1 MAPK pathways (Park et al. 2006; Zhou et al. 2014). To determine the effect of the dominant active form of Ras2 on its interaction with Mst11, we generated the Ras $2^{\mathrm{G} 18 \mathrm{~V}}$ (Zhou et al. 2014) prey construct pHZ109 and cotransformed it with the Mst11 bait construct pGP80 into the yeast strain YRG2. The resulting Trp+ Leu+ transformants had more robust growth on SD-Leu-TrpHis (SD-His) plates (Fig. 4A) and stronger $\beta$-galactosidase activities (Fig. 4B) than the yeast transformant containing pGP80 and the wild-type Ras2 prey (Zhao et al. 2005). These results indicated that the $\mathrm{G} 18 \mathrm{~V}$ mutation enhances the interaction of Ras 2 with Mst11. Because $R A S 2^{\mathrm{G} 18 \mathrm{~V}}$ was reported to be a dominant active allele (Zhou et al. 2014), Mst11 may have a stronger affinity for the active Ras2-GTP form in M. oryzae.

\section{Deletion of the RA domain has no obvious effect on the activation of Pmk1, Mps1, or Osm1.}

To determine whether the RA domain of Mst11 is important for Pmk1 activation, we assayed the phosphorylation of MAPKs at the TEY dual phosphorylation site. When detected with an anti-TpEY-specific antibody, the Pmk1 (42-kDa) band was not detectable in total proteins isolated from the mst11 mutant, indicating that Pmk1 activation is blocked. In wild-type strain 70-15 and transformant MRD-2, phosphorylation of Pmk1 was detectable in proteins isolated from vegetative hyphae (Fig. 5A). The level of Pmk1 phosphorylation was not significantly affected in the $M S T 11^{\Delta \mathrm{RA}}$ transformant MRD-2. The phosphorylation of Mps1, the only other MAPK with the TEY site in M. oryzae (Xu et al. 1998), also was similar between the wild type and transformant MRD-2 (Fig. 5A). These results indicate that the RA domain of Mst11 is not essential for the activation of Pmk1 or Mps1.

In the budding yeast, Ste11 is involved in both the pheromone response and high-osmolarity glycerol pathways. Therefore, we determined the effect of RA domain deletion on

Table 2. Assays for appressorium formation, penetration, and virulence in Magnaporthe oryzae strains ${ }^{\mathbf{v}}$

\begin{tabular}{lcccc}
\hline Strain & Branched germ tubes (\%) $^{\mathbf{w}}$ & Two appressoria (\%) $^{\mathbf{x}}$ & Penetration efficiency (\%) $^{\mathbf{y}}$ & Lesions/5-cm leaf tip) $^{\mathbf{z}}$ \\
\hline $70-15(\mathrm{WT})$ & $2.8 \pm 0.4 \mathrm{~b}$ & $2.3 \pm 0.5 \mathrm{c}$ & $68.7 \pm 3.2 \mathrm{a}$ & $47.5 \pm 3.2 \mathrm{a}$ \\
yk86 (mst11) & $0 \mathrm{c}$ & $0 \mathrm{~d}$ & $0 \mathrm{~d}$ & $0 \mathrm{~d}$ \\
Yk86C (mst11/MST11) & $3.7 \pm 2.3 \mathrm{~b}$ & $4.3 \pm 1.7 \mathrm{bc}$ & $59.8 \pm 5.2 \mathrm{~b}$ & $40.7 \pm 5.3 \mathrm{~b}$ \\
MRD-2 (mst11/MST11 ${ }^{\Delta \mathrm{RA})}$ & $70.5 \pm 3.5 \mathrm{a}$ & $50.7 \pm 3.8 \mathrm{a}$ & $30.5 \pm 2.5 \mathrm{c}$ & $31.5 \pm 3.0 \mathrm{c}$ \\
\hline
\end{tabular}

\footnotetext{
${ }^{\mathrm{v}}$ Means and standard errors were estimated from at least three independent experiments. Data were analyzed with Duncan's pair-wise comparison. Different letters mark statistically significant differences $(P=0.05)$.

${ }^{\mathrm{w}}$ Percentage of germ tubes that branched after the formation of an apical appressorium on the hydrophobic plastic coverslips at $24 \mathrm{~h}$.

${ }^{x}$ Percentage of branched germ tubes that formed two appressoria by $48 \mathrm{~h}$.

${ }^{\mathrm{y}}$ Percentage of appressoria that penetrated and formed infectious hyphae in onion epidermal cells by $48 \mathrm{~h}$

${ }^{\mathrm{z}}$ Number of lesions formed on rice leaves sprayed with $2 \times 10^{4}$ conidia/ml at 7 days postinoculation.
} 
the phosphorylation of the Osm1 MAPK (Dixon et al. 1999) with an anti-TpGY antibody. Osm1 phosphorylation was similar among 70-15, yk86, and MRD-2 in vegetative hyphae harvested from $5 \times$ YEG (per milliliter, $5 \mathrm{mg}$ yeast extract and $10 \mathrm{mg}$ glucose) cultures (Fig. 5B). However, when treated with $0.7 \mathrm{M} \mathrm{NaCl}$, the phosphorylation level of Osm1 increased in 70-15 and MRD-2 but not in the mst11 deletion mutant (Fig. 5B). These data indicated that Mst11 is involved in crosstalking with the Osm1 pathway in M. oryzae but the RA domain is not important for the role of Mst11 in Osm1 activation.

The middle region of $M S T 11$ is essential for its function.

Because the RA domain is not essential for Pmk1 activation and appressorium formation, we generated the MST11 mutation allele that was deleted of the entire middle region (amino acid residues 263 to 565 ) between the $\mathrm{N}$-terminal SAM and C-terminal protein kinase domains (Fig. 6A). In the mst11/MST11 $1^{\Delta \mathrm{MR}}$ transformants, appressorium formation was not observed, although they still grew deformed germ tubes on hydrophobic surfaces (Fig. 6B). Similar to the mst11 deletion mutant, the mst11/MST1 $1^{\Delta \mathrm{MR}}$ transformant was nonpathogenic in infection assays with rice seedlings (Fig. 6C). These data indicate that the middle region of MST11 is essential for appressorium formation and plant infection. It is possible that this middle region between the SAM and kinase domains plays a modulation or regulatory role in Mst11 activation.
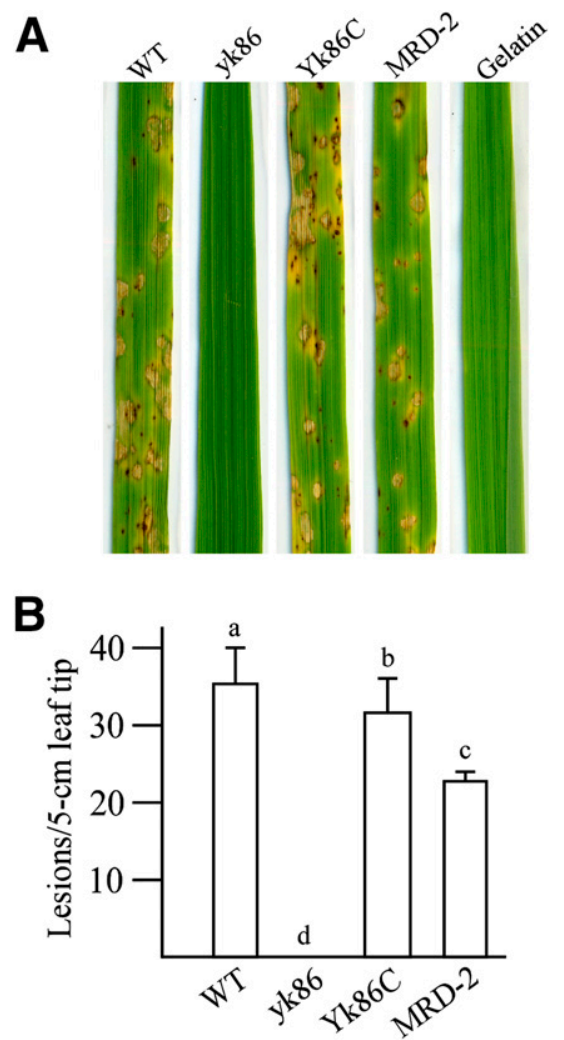

Fig. 2. Infection assays with 2 -week-old rice seedling. A, Leaves were sprayed with conidial suspensions $\left(5 \times 10^{4}\right.$ conidia/ml $)$ of $70-15$, the $m s t 11$ mutant yk86, complemented strain Yk86C, and MRD-2. Inoculation with $0.25 \%$ gelatin was used as the negative control. B, The number of blast lesions per $5-\mathrm{cm}$ leaf tip caused by the same set of strains was quantified as described (Kershaw et al. 1998). Data from three independent replicates were analyzed with the GLM procedure of SAS (SAS Institute Inc., Cary, NC, U.S.A.). Different letters mark statistically significant differences $(P=$ $0.05)$.
Two conserved phosphorylation sites play a role in Mst11 activation.

Sequence alignment analysis of the middle region of Mst11 and its orthologs from other fungi showed that two of the three PAK phosphorylation sites that have been identified in Ste11 (van Drogen et al. 2000) are conserved in Mst11 orthologs

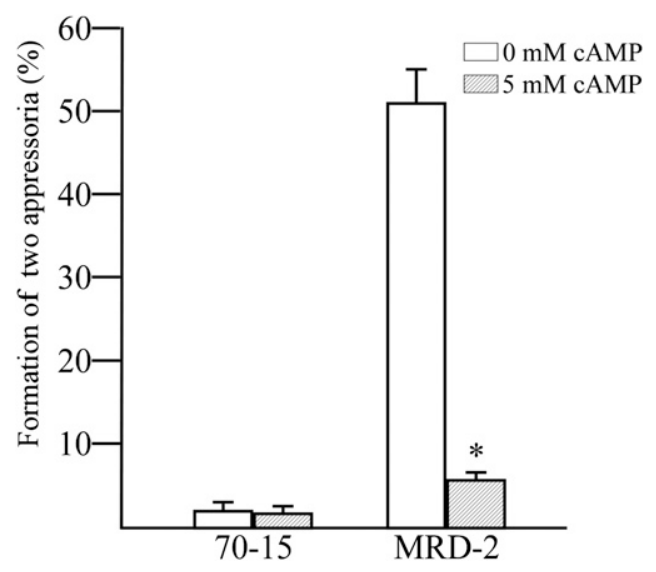

Fig. 3. Assays for the effect of exogenous cAMP on the $m s t 11 / M S T 11^{\triangle \mathrm{RA}}$ transformant MRD-2. Conidia of 70-15 and strain MRD-2 were assayed for appressorium formation on the hydrophobic surface of GelBond membranes with or without $5 \mathrm{mM}$ cAMP after incubation for $48 \mathrm{~h}$. Mean and standard deviation were calculated with results from three replicates. An asterisk (*) indicates statistically significant differences between transformant MRD-2 treated with and without $5 \mathrm{mM}$ cAMP $(P=0.05)$. Exogenous cAMP partially suppressed the formation of two appressoria on branched germ tubes.

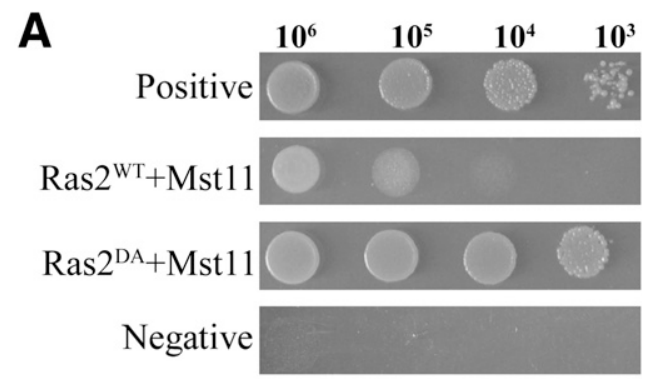

B

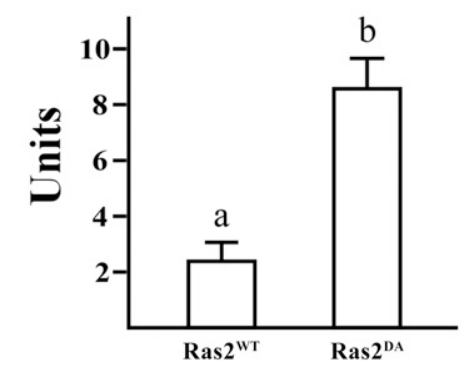

Fig. 4. Yeast two-hybrid assays for the effect of the G18V mutation in Ras2 on its interaction with Mst11. A, Yeast cells of marked concentrations (cells per milliliter) expressing the Mst11 prey and Ras2 (Ras ${ }^{\mathrm{WT}}$ ) or Ras2 $2^{\mathrm{G} 18 \mathrm{~V}}$ $\left(\operatorname{Ras}^{\mathrm{DA}}\right)$ bait constructs were assayed for growth on SD-His plates. The Mst50-Mst11 and Mst50-Pmk1 interactions (Zhao et al. 2005) were used as positive and negative controls, respectively. B, Quantitative assays of $\beta$-galactosidase activities with yeast-cell yeast transformants expressing the Mst11 prey and Ras2 (Ras ${ }^{\mathrm{WT}}$ ) or Ras $2^{\mathrm{G} 18 \mathrm{~V}}\left(\operatorname{Ras}^{\mathrm{DA}}\right)$ bait constructs. One unit of $\beta$-galactosidase is defined as the enzyme activity required to hydrolyze $1 \mathrm{mmol}$ of $o$-nitrophenyl- $\beta$-D-galactopyranoside to $o$-nitrophenol and D-galactose per minute. Means and standard deviations were calculated from three independent experiments. Different letters mark statistically significant differences by the GLM procedure of SAS (SAS Institute Inc., Cary, NC, U.S.A.) $(P=0.05)$. 
(S453 and S458; Supplementary Fig. S2). Although PAKs are dispensable for appressorium formation and Pmk1 activation ( $\mathrm{Li}$ et al. 2004), it remains possible that these two putative phosphorylation sites play a role in Mst11 activation. To determine their functions, we generated the $M S T 11^{\triangle \mathrm{PP}}$ allele deleted of residues 450 to 458 (Fig. 6A) and transformed it into mutant yk86. The resulting mst11/MST1 $1^{\Delta \mathrm{PP}}$ transformant PH29 still formed appressoria but at a reduced efficiency. Only $13.5 \% \pm 4.0 \%$ of $\mathrm{PH} 29$ germ tubes developed appressoria on hydrophobic surfaces by $24 \mathrm{~h}$. Under the same conditions, over $90 \%$ of wild-type germ tubes produced appressoria (Fig. 6A; Table 3). Similar to the pmkl and mst11 mutants, most of the germ tubes of the MST1 $1^{\triangle \mathrm{PP}}$ transformant had deformed tips on hydrophobic surfaces (Fig. 6B), suggesting that residues 450 to 458 , including S453 and S458, are dispensable for surface recognition but important for appressorium formation.

In spray-infection assays with 2-week-old rice seedlings of cultivar Nipponbare, PH29 mutant was nonpathogenic (Fig. 6C). No typical blast lesions were observed. To determine the defects of transformant PH29 in plant infection, we assayed appressorium formation and invasive growth with barley epidermal cells. On barley leaves, only approximately $10 \%$ of $\mathrm{PH} 29$ germ tubes formed appressoria, which is consistent with its defects in appressorium formation on hydrophobic surfaces (Table 3). In addition, invasive hyphae formed by PH29 inside barley epidermal cells had limited growth in comparison with those of the wild-type strain (Fig. 6D). These results suggested that phosphorylation at S453 and S458 is also important for invasive growth in plant cells.

\section{Mutations at S453 and S458 of Mst11 affect its function in appressorium morphogenesis.}

To further characterize the function of these two putative phosphorylation sites, we constructed the $M S T 11^{\text {S453D S458D }}$ mutant allele and transformed it into the mst11 mutant yk86. On hydrophobic surfaces, the mst11/MST1 $1^{\mathrm{S} 453 \mathrm{D}} \mathrm{S458 \textrm {D }}$ transformant PD65 was normal in conidium germination but only $12.7 \% \pm$ $2.9 \%$ of its germ tubes formed appressoria. In comparison with the wild type, transformant PD65 was significantly reduced in the efficiency of appressorium formation (Fig. 7A; Table 3). In penetration assays with barley leaf epidemic cells, appressorium formation was reduced, but successful penetration and development of invasive hyphae were not observed in the mst11/MST11 ${ }^{\text {S453D S458D }}$ transformant PD65 (Fig. 7B). When 2week-old seedlings of rice cultivar Nipponbare were sprayed with conidia from wild-type strain 70-15, numerous blast lesions were observed 7 dpi. Transformant PD65 failed to cause typical blast lesions on rice leaves (Fig. 7C). These data suggested that the putative phosphorylation sites S453 and S458 are important for appressorium formation and essential for the function of MST11 in appressorial penetration and pathogenesis. However, the S453D and S458D mutations resulted in defects in appressorium formation and pathogenicity, which is different from expected effects of dominant active mutations of MST11 on formation of appressoria on hydrophilic surface.

\section{The N-terminal region of Mst11 binds} to its kinase domain.

Although the PAKs appear to be dispensable for the activation of the Mst11-Mst7-Pmk1 cascade (Li et al. 2004), we found that S453 and S458 are important for Mst11 function. Therefore, it is possible that, like Ste11 in the budding yeast (van Drogen et al. 2000), the N-terminal region of Mst11 negatively interacts with its kinase domain. To test this hypothesis, we cotransformed the prey construct of Mst11 ${ }^{585-913}$ (pYK50) that mainly contains the C-terminal kinase domain with the bait construct of Mst11 ${ }^{1-582}$ (pYK55) into the yeast strain YRG2. The resulting yeast transformants expressing these constructs were able to grow on SD-His plates and had $\beta$-galactosidase activities (Fig. 8), indicating that the $\mathrm{N}$-terminal region of Mst11 directly interacts with its kinase domain. Therefore, it is likely that Mst11 has self-inhibitory binding in $M$. oryzae. Conformational changes in the middle region (regulatory function), such as binding to activated Ras2 or phosphorylation at S453 and S458, may relieve this binding and activate Mst11.

S789 of Mst11 is important for its self-inhibitory binding.

In serine/threonine protein kinases, the DFG motif is located at the beginning of the activation loop and plays an important role in the regulation of kinase activity (Treiber and Shah 2013). In yeast, the T596I mutation next to the DFG motif in STE11-4 has a dominant active effect (Stevenson et al. 1992). In Mst11, S789 is right next to the DFG motif (residues 790 to 792). To determine the importance of S789 in the self-binding of Mst11, we introduced the S789G mutation into the prey construct of Mst11 $1^{585-913}$ to generate pYK58. In yeast twohybrid assays, the transformants harboring pYK55 and pYK58 had weaker growth on SD-His and $\beta$-galactosidase activities compared with the pYK55 and pYK50 transformants (Fig. 8). These data suggest that the $\mathrm{S} 789 \mathrm{G}$ mutation reduced the interaction between the $\mathrm{N}$-terminal region of Mst11 and its kinase domain.

\section{The S789G mutation resulted}

in gain-of-function activities in MST11.

To determine the effect of $\mathrm{S} 789 \mathrm{G}$ mutation on the function or activation of Mst11, we generated the MST11 ${ }^{\mathrm{S} 789 \mathrm{G}}$ allele (pYK54) and transformed it into the wild-type strain. Transformant WY54 was confirmed to have a single copy of ectopically integrated pYK54 (Table 1). Whereas it was normal in appressorium formation on hydrophobic surfaces, transformant WY54 also efficiently formed appressoria on the hydrophilic side of GelBond membranes (Fig. 9A). By 24 h, approximately $52.5 \% \pm 4.6 \%$ of germ tubes produced appressoria on hydrophilic surfaces in the MST11 $1^{\mathrm{S} 789 \mathrm{G}}$ transformant (Table 3).
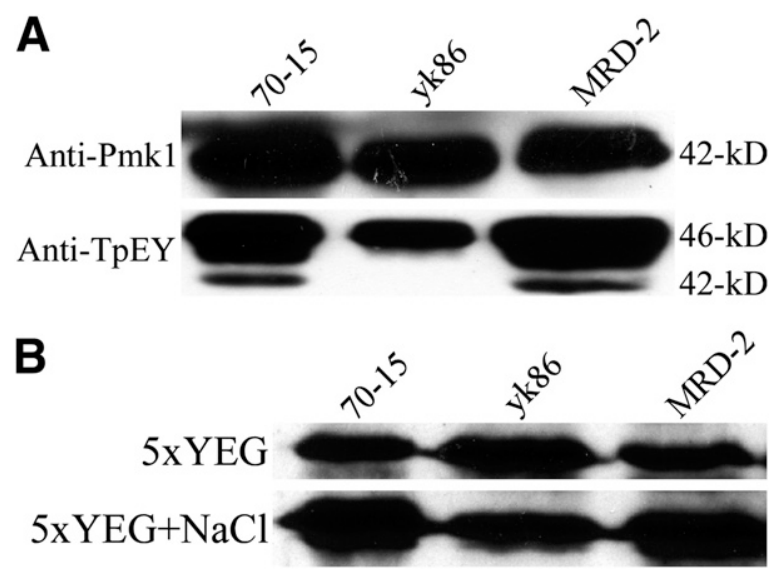

Fig. 5. Assays for the phosphorylation of Pmk1, Mps1, and Osm1 mitogenactivated protein kinases (MAPKs). A, Western blot analyses with total proteins isolated from vegetative hyphae of the wild-type strain 70-15, mst11 mutant yk86, and MST1 $1^{\triangle \mathrm{RA}}$ transformant MRD-2. When detected with an anti-Pmk1 antibody (top panel), the 42-kDa Pmk1 band was observed in total proteins isolated from all three strains. However, an anti-TpEY antibody detected the 42-kDa Pmk1 band only in 70-15 and transformant MRD-2. The phosphorylation of Mps1 (46-kDa band) was detectable in all these strains. B, The phosphorylation of Osm1 was detected with an anti-TpGY specific antibody by Western blot analysis. Total proteins were isolated from vegetative hyphae harvested from $5 \times$ YEG (per milliliter, $5 \mathrm{mg}$ yeast extract and $10 \mathrm{mg}$ glucose) cultures of 70-15, yk86, and MRD-2 treated with or without $0.7 \mathrm{M} \mathrm{NaCl}$. 
Therefore, the S789G mutation had a dominant active effect on appressorium formation, possibly by interfering with the selfinhibitory binding between the N-terminal region of Mst11 with its kinase domain.

To further confirm this observation, we assayed Pmk1 activation in transformant WY54 with the anti-TpEY antibody. In comparison with wild-type strain 70-15, the Pmk1 phosphorylation level was significantly increased in transformant WY54 (Fig. 9B), indicating that the S789G mutation may also decrease the self-inhibitory binding of Mst11 in vivo. Nevertheless, in infection assays with 2-week-old seedlings of rice cultivar Nipponbare, blast lesions were observed on leaves sprayed with WY54 conidia (Fig. 9C). In comparison with wild type, no obvious changes in virulence were observed in transformant WY54. Therefore, the S789G mutation had no significant effect on virulence.

\section{Expression of $M S T 11^{\text {S789G }}$ failed to suppress the defects of mst50 mutants.}

We also introduced the $M S T 11^{\mathrm{S} 789 \mathrm{G}}$ allele into the $m s t 50$ mutant MF103 (Park et al. 2006) to determine its function. Like the mst50 or pmkl mutant, the resulting mst50/MST11 ${ }^{\mathrm{S} 789 \mathrm{G}}$ transformant MC04 (Table 1) failed to form appressoria on hydrophobic surfaces (Fig. 10A). In infection assays, it was nonpathogenic (Fig. 10B). Therefore, expression of the MST1 $^{\mathrm{S} 789 \mathrm{G}}$ allele is not sufficient to suppress the defects of the mst50 mutant in appressorium formation and plant infection. One likely explanation is that MST50 is known to function as an adaptor protein that interacts with both Mst11 and Mst7 (Park et al. 2006). In the absence of Mst50, the interaction between Mst11 and Mst7 may be too weak or transient, if not blocked. In addition, Mst50 is known to be involved in other signaling pathways.

Because of its inhibitory binding to the kinase domain, we generated the MSTI1 $1^{\Delta 6-583}$ allele and transformed it into the mst11 deletion mutant. The resulting transformant NT48 expressing the $M S T 11^{\Delta 6-583}$ allele failed to form appressoria on hydrophobic surfaces (Fig. 10A) and was nonpathogenic in infection assays with rice or barley seedlings (Fig. 10B), indicating that deletion of the N-terminal region had no gain-offunction effect in $M$. oryzae.

\section{DISCUSSION}

The Mst11 MEK kinase plays a key role in the activation of the Pmk1 MAPK cascade and infection-related morphogenesis

Table 3. Appressorium formation assays with strains expressing different MST11 alleles

\begin{tabular}{|c|c|c|c|}
\hline \multirow[b]{2}{*}{ Strain } & \multirow[b]{2}{*}{ Genotype } & \multicolumn{2}{|c|}{ Appressorium formation $(\%)^{\mathbf{z}}$} \\
\hline & & Hydrophobic & Hydrophillic \\
\hline $70-15$ & Wild type & $92.7 \pm 2.1 \mathrm{a}$ & $14.0 \pm 1.3 \mathrm{~b}$ \\
\hline WY54 & $M S T 11^{\mathrm{S} 789 \mathrm{G}} / 70-15$ & $87.9 \pm 3.8 \mathrm{a}$ & $52.5 \pm 4.6 \mathrm{a}$ \\
\hline RP37 & mst11/MST11 $\mathrm{MMR}$ & $0 \mathrm{c}$ & NA \\
\hline PH29 & $m s t 11 / M S T 11^{\Delta \mathrm{PP}}$ & $13.5 \pm 4.0 \mathrm{~b}$ & $0 \mathrm{c}$ \\
\hline PD65 & $m s t 11 / M S T 11^{\text {S453D S458D }}$ & $12.7 \pm 2.9 b$ & $0 \mathrm{c}$ \\
\hline
\end{tabular}

${ }^{\mathrm{z}}$ Percentage of germ tubes that formed appressoria on the hydrophobic or hydrophilic side of GelBond membranes by $24 \mathrm{~h}$. Means and standard errors were estimated from at least three independent experiments. Data were analyzed with Duncan's pair-wise comparison. Different letters mark statistically significant differences $(P=0.05)$.
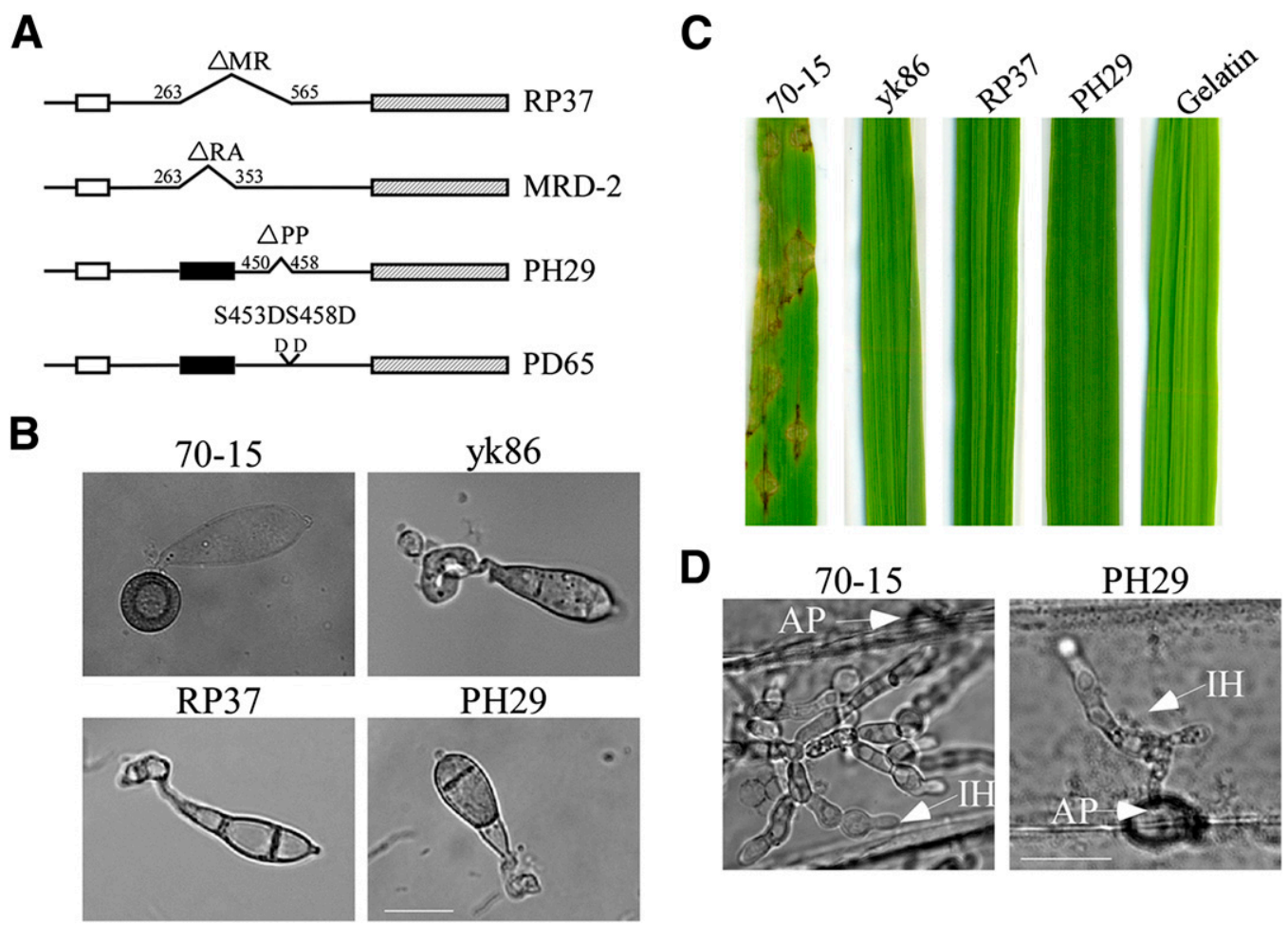

Fig. 6. Generation and functional characterization of different MST11 mutant alleles. A, Schematic drawing of the MST11 gene showing the Ras-association (RA) domain (black box) and two putative phosphorylation sites (S453 and S458) between the sterile alpha motif and kinase domains. The RA domain and middle region (263 to 565) were deleted in the MST1 $1^{\triangle \mathrm{RA}}$ and MST1 $1^{\Delta \mathrm{MR}}$ constructs. S453 and S458 were deleted in $M S T 11^{\triangle \mathrm{PP}(453-458)}$ or changed to aspartic acids in MST11 S453D S458D. All these constructs had the same MST11 promoter as in the complementation construct pYK35. B, Appressorium formation assays with the wild-type strain 70-15, mst11 mutant yk86, and transformants of yk86 expressing the MST11 ${ }^{\Delta \mathrm{MR}}$ (RP37) or MST1 ${ }^{\triangle \mathrm{PP}}$ (PH29) allele on artificial hydrophobic surfaces. C, Rice leaves were sprayed with conidia of 70-15, yk86, RP37, and PH29. Inoculation with $0.25 \%$ gelatin was used as the negative control. D, Penetration assays with barley epidermal cells with 70-15 and transformant PH29. Invasive hyphae formed by PH29 had limited growth in barley leaf cells. AP, appressoria and IH, invasive hyphae. 
in $M$. oryzae (Zhao et al. 2005). In addition to the N-terminal SAM, Mst11 also has a RA domain that is well conserved among its orthologs from filamentous fungi. Mst11 directly interacts with Ras2 that functions upstream from both cAMP signaling and Pmk1 MAPK pathways (Zhou et al. 2014). In this

A
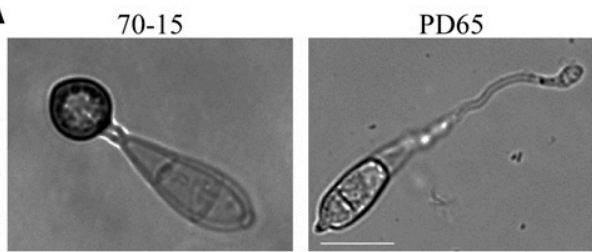

B

$70-15$
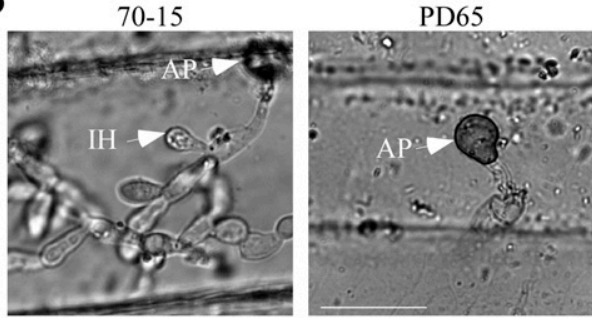

C

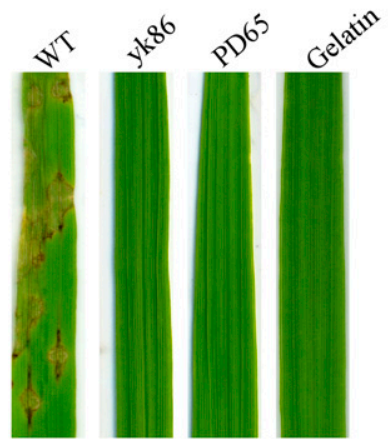

Fig. 7. Appressorium formation and infection assays with the wild-type strain 70-15 and mst 1 1/MST11 ${ }^{\mathrm{S} 453 \mathrm{DS} 458 \mathrm{D}}$ transformant PD65. A, Conidia of 70-15 and transformant PD65 were incubated on hydrophobic surfaces for $24 \mathrm{~h}$. Bar $=101 \mathrm{~m}$. B, Penetration assays with barley epidermal cells. Rare appressoria formed by transformant PD65 failed to penetrate epidermal cells of barley leaves. AP, appressoria and $\mathrm{IH}$, invasive hyphae. C, Rice leaves were sprayed with conidia of 70-15, mst11 deletion mutant yk86, and transformant PD65. Inoculation with $0.25 \%$ gelatin was used as a negative control. Typical leaves were photographed 7 days postinoculation.

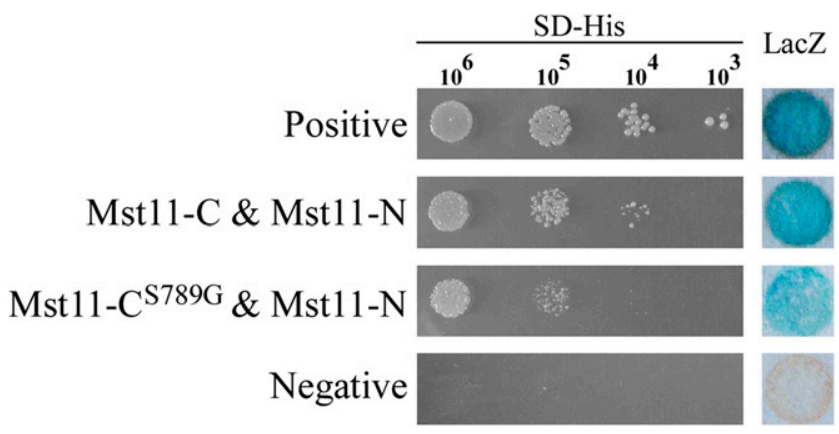

Fig. 8. Yeast two-hybrid assays for the interaction of the $\mathrm{N}$-terminal region of Mst11 with its kinase domain. Yeast cells of marked concentrations (cells per milliliter) expressing the prey construct of the $\mathrm{N}$-terminal region (residues 1 to 582, Mst11-N) and bait construct of its kinase domain (residues 585 to 913 ) without (Mst11-C) or with the S789G mutation (Mst11-C $\mathrm{C}^{\mathrm{S789G}}$ ) were assayed for growth SD-Leu-Trp-His (SD-His) plates. The positive and negative controls were provided in the HybridZap2.1 XR library construction kit. The same set of yeast strains were assayed for $\beta$-galactosidase activities (LacZ). study, we found that the G18V mutation of Ras2 (Zhou et al. 2014) enhanced its interaction with Mst11, suggesting that Mst11 may have increased affinity for the activated form of Ras2. It is possible that interacting with Ras2-GTP is related to the activation of Mst11 during appressorium formation. However, deletion of the RA domain in MST11 did not block appressorium formation. In the $M$. oryzae genome, both Mst11 and Mst50 contain the RA domain and they interact with each other via the SAM domains (Park et al. 2006; Zhao et al. 2005). It is possible that the Mst50-Ras2 interaction is partially redundant with the Mst11-Ras2 interaction during appressorium formation.

In a previous study, expression of the $M S T 11^{\triangle \mathrm{RA}}$ allele in the mst11 mutant was reported to rescue its defects in appressorium formation (Zhao et al. 2005). Close examination in this study showed that, after the formation of melanized appressoria at the tip, germ tubes of the $M S T 11^{\triangle \mathrm{RA}}$ transformant MRD-2 often branched to produce another appressoria by $48 \mathrm{~h}$, which was not observed in a previous study (Zhao et al. 2005). In the wild type, the formation of a terminal appressorium triggers cell-death events in conidium compartments and germ tubes

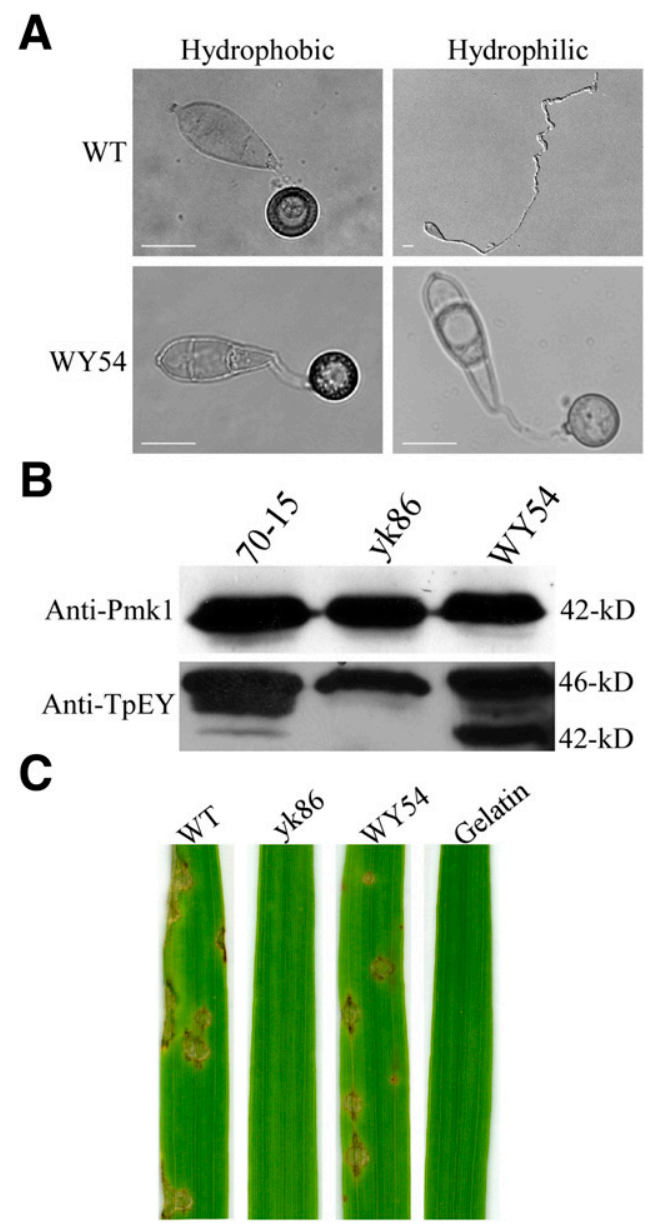

Fig. 9. Assays for the effects of expressing of the $M S T 11^{\mathrm{S} 789 \mathrm{G}}$ allele in the wild-type stain 70-15. A, Appressorium formation assays on hydrophobic or hydrophilic surfaces with conidia of the wild-type strain 70-15 and its $M S T 11^{\mathrm{S} 789 \mathrm{G}}$ transformant WY54. Bar $=101 \mathrm{~m}$. B, Protein gel blot analysis of the expression and activation of Pmk1. Total proteins were isolated from vegetative hyphae of 70-15, mst11 mutant yk86, and transformant WY54. When detected with an anti-TpEY antibody (bottom), a 42-kDa band was detected in proteins isolated from 70-15 and WY54 but not in yk86. Detection with the anti-Pmklantibody (top) showed that similar amounts of proteins were loaded in each lane. $\mathbf{C}$, Rice leaves were sprayed with conidia of the same set of strains. Inoculation with $0.25 \%$ gelatin was used as the negative control. 
(Kershaw and Talbot 2009; Veneault-Fourrey et al. 2006). Further growth and branching of germ tubes must be inhibited after the initiation of appressorium formation. Because exogenous cAMP suppressed the defects of strain MRD-2 in germtube branching (Fig. 3), it is likely that improper regulation of germ-tube growth and appressorium formation is partially affected by the cAMP pathway. The association of Mst11 with Ras 2 may be important for signaling the arrest of germ-tube growth after the tip beginning to differentiate on hydrophobic surfaces. Therefore, the cross-talking between the cAMP signaling and Pmk1 MAPK pathways may occur at the binding of Ras2 to Mst11. It is possible that the RA domain of Mst11 is involved in the feedback regulation of the Ras-cAMP signaling pathway during appressorium formation and triggering cell death in conidia (Veneault-Fourrey et al. 2006).

Transformant MRD-2 was reduced in appressorial penetration and virulence (Table 2). Because turgor generation in appressoria requires the utilization of carbohydrate reserves in conidia (Thines et al. 2000), formation of multiple appressoria likely affects appressorium turgor generation. Therefore, reduced penetration efficiency and virulence of the $M S T 11^{\triangle \mathrm{RA}}$ transformant may be directly related to its defects in the arrest of germ-tube growth after appressorium formation and generation of normal appressorium turgor. The observation of reduced virulence in the MST11 ${ }^{\triangle \mathrm{RA}}$ transformant was slightly different from the earlier report (Zhao et al. 2005), which may be related to differences in infection conditions. The reduction of strain MRD-2 in virulence is relatively minor and the earlier study did not count lesion numbers and quantitatively assay changes in virulence. In addition, when sprayed at a higher conidium concentration, as in the previous study (Zhao et al. 2005), it might be difficult to detect subtle changes in virulence caused by RA domain deletion. In Ustilago maydis, transformants expressing the $k p p 4^{\mathrm{K} 481 \mathrm{E}}$ allele with the point mutation in the RA domain also were reduced in virulence (Müller et al. 2003), suggesting that the RA domain of this kinase plays a conserved role in virulence in plant-pathogenic fungi.

Although the RA domain is not essential, we found that deletion of the middle region (residues 263 to 565) blocked appressorium formation and the activation of the Pmk1

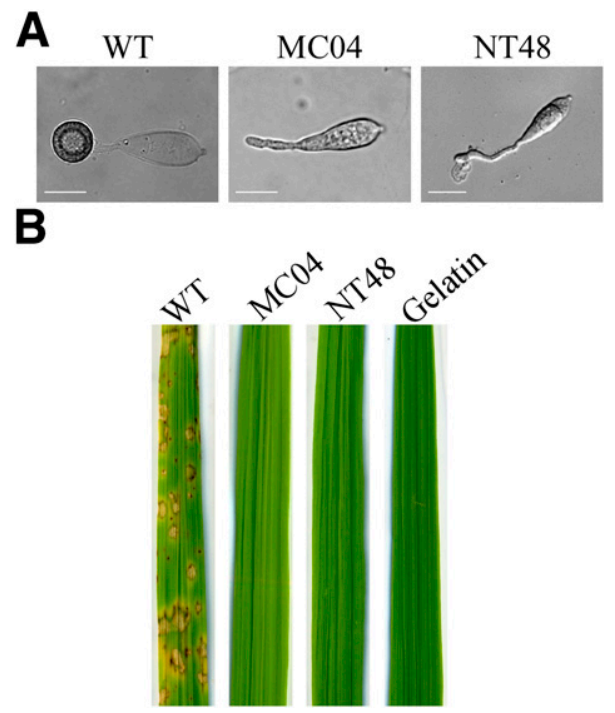

Fig. 10. Appressorium formation and infection assays with the mst50 MST11 $^{\text {S789G }}$ transformant MC04. (a). Conidia of the wild-type strain 70-15, NT48 (MST11 $1^{\Delta 6-583}$ transformant), and MC04 were incubated on plastic coverslips for 24 h. (b). Rice seedlings were inoculated with 70-15, NT48, and MC04 and examined for lesion developments 7 dpi. Inoculation with $0.25 \%$ gelatin was used as a negative control. cascade. In Saccharomyces cerevisiae, Ste 11 is activated by the PAK Ste 20 by phosphorylation at serine residues 302,306 , and 307 (van Drogen et al. 2000). Sequence alignment showed that S306 is not conserved in its orthologs from M. oryzae and other fungi, although it plays a more critical role in Ste11 activation in yeast (van Drogen et al. 2000). For the other two phosphorylation sites, S302 and S307 of Ste11 are equivalent to S453 and S458 of Mst11. In M. oryzae, deletion of residues 450 to 458 resulted in defects in appressorium formation and loss of virulence. Furthermore, the mst11/MST11 $1^{\mathrm{S} 453 \mathrm{D}}$ S458D transformant PD65 was nonpathogenic and defective in appressorium formation and penetration. Therefore, S453 and S458 must play critical roles in Mst11 activation or function. Most likely, S453 is more important than S458, because sequence alignment showed that only S453 is well-conserved in Mst11 and its orthologs from other filamentous fungi. The residues at the S458 position are conserved in Mst11 orthologs from Sordariomycetes but not in those from other fungi. Interestingly, the S453D and S458D mutations did not result in a putative dominant active MST11 allele. However, it is wellknown that changes from serine/threonine to aspartic acid residues do not always result in gain-of-function mutations (Bauer et al. 2003). In M. oryzae, the S453D and S458D mutations appeared to negatively impact the function of Mst11 or its activation.

In M. oryzae, MST20, the ortholog of yeast STE20, is dispensable for appressorium formation and plant infection $(\mathrm{Li}$ et al. 2004). In fact, the mst20 mutant had no detectable phenotype except a minor reduction in conidiation, suggesting that MST20 is not involved in the activation of Mst11-Mst7-Pmk1 cascade. CHM1, the only other PAK gene, also is not essential for appressorium formation and Pmk1 activation, although it has pleiotropic defects and is nonpathogenic ( $\mathrm{Li}$ et al. 2004). In Fusarium graminearum, the Ste 20 ortholog also is dispensable for virulence (Wang et al. 2011). In Ustilago maydis, the Smu1 PAK is not essential but is important for normal virulence and mating, although its role in the pheromone response MAPK pathway is not known (Smith et al. 2004). Therefore, the activation of Mst11 may require phosphorylation of S453 and S458 by unknown protein kinases that have not been identified. Nevertheless, the chml mst20 double mutant seemed to be lethal ( $\mathrm{Li}$ et al. 2004) and it remains possible that Mst20 and Chm1 have redundant functions in the activation of Mst11 by phosphorylation of one or both S453 and S458. Another possibility is that Mst11 may have self-phosphorylation activities when its C-terminal catalytic kinase domain becomes active after being released from the self-inhibitory binding with the $\mathrm{N}$-terminal region.

The self-inhibitory binding between the N-terminal and C-terminal regions have been observed in different protein kinases (Gustchina et al. 2002; Shewchuk et al. 2000). In yeast two-hybrid assays, the N-terminal region of Mst11 directly interacted with its kinase domain, which could be reduced or relinquished by the $\mathrm{S789G}$ mutation next to the DFG motif in the catalytic domain. In M. oryzae, MST11 $1^{\mathrm{S} 789 \mathrm{G}}$ appeared to function as a dominant active allele to stimulate appressorium formation on hydrophilic surfaces. It is possible that the selfinhibitory binding may be reduced or relinquished in transformant WY54, which may result in a constitutive active Mst11 MEK kinase. Because some kinase inhibitors exhibit their inhibitory effects by interacting with the DFG triplet (Peng et al. 2013), the N-terminal region of Mst11 may negatively regulate its kinase domain through the same mechanism. In addition, based on the predicted three-dimensional structure of Mst11, according to its orthologs, the S789G mutation likely results in binding between magnesium and residue D790 and the formation of a hydrogen bond between 
D790 and G792 (Supplementary Fig. S3), which is the active form of the DFP motif (Steichen et al. 2012). However, unlike the dominant active $M S T 7$ allele (Zhao et al. 2005), expression of the MST1 $11^{\mathrm{S} 789 \mathrm{G}}$ allele in the mst50 mutant failed to suppress its defects in appressorium formation and plant penetration. One possible explanation is that Mst50 is an adaptor protein (Park et al. 2006) and the absence of Mst50 diminished or reduced the Mst11-Mst7 interaction. Expression of a dominant active allele of MST11 in the absence of Mst50 is not sufficient to activate Mst7 by Mst11 because they were not brought together by the adaptor protein.

Taken together, our data suggest that Mst11 is activated by releasing from the self-inhibitory binding between the $\mathrm{N}$-terminal region and the kinase domain, and the activation of Mst11 is mediated by the regulatory sequences in the middle region. It is possible that phosphorylation at one or both S453 and S458 plays a critical role in the release of this inhibitory interaction of Mst11. However, the binding of activated Ras2 to the RA domain of Mst11 may also cause conformational changes and affect the interaction of the $\mathrm{N}$-terminal region with its kinase domain. Ras2 also interacts with Mst11 and Mst50, which is known to interact with both Mst11 and Mst7 (Park et al. 2006). Mac1, the adenylate cyclase, is the key component of the cAMP pathway, which plays a role in surface recognition and appressorium morphogenesis in $M$. oryzae (Choi and Dean 1997). The activation of the Mst11-Mst7-Pmk1 MAPK cascade may result in a feedback activation of Mac1 adenylate cyclase, which, in turn, prevents germ-tube branching and formation of additional appressoria on branched germ tubes.

\section{MATERIALS AND METHODS}

Strains and culture conditions.

The wild-type $M$. oryzae strains, the MRD-2 and MRD-5 mutants (Zhao et al. 2005), and all the transformants generated in this study (Table 1) were cultured on oatmeal agar plates as previously described (Ding et al. 2010; Xu and Hamer, 1996). Mycelia harvested from 2-day-old liquid complete medium or $5 \times$ YEG cultures shaken at $150 \mathrm{rpm}$ at $25^{\circ} \mathrm{C}$ were used for isolating DNA, proteins, and protoplasts (Bruno et al. 2004; Xue et al. 2002). For fungal transformation, culture media were supplemented with $2501 \mathrm{~g}$ of hygromycin B per milliliter (Calbiochem, La Jolla, CA, U.S.A.) or $2501 \mathrm{~g}$ of zeocin per milliliter (Invitrogen, Carlsbad, CA, U.S.A.) to select for hygromycin- or zeocin-resistant transformants.

\section{Generation of domain deletion constructs and mutant alleles of MST11.}

To delete the two putative phosphorylation sites, a 1.4-kb fragment including the promoter and N-terminal portion of MST11 was amplified with primer pair SRF1 and PHR2 and was cloned between the ApaI and ClaI sites on pYK11 (Zhao et al. 2005) as pYK26. A 1.7-kb downstream fragment of MST11 was amplified with primer pair PHF1 and SRR2 and was cloned between the ClaI and SpeI sites on pYK26 as pYK29 (Fig. 5A). To generate the $M S T 11^{\Delta 253-565}$ construct pYK37 (Fig. 5A), a 1.9-kb upstream fragment of MST11 was amplified with primer pair NSF2 and SRR1 and was ligated with the downstream fragment amplified with primer pair PHF1 and SRR2. Primers MSDF1 and MSDR1 were used to introduce the S453D S458D mutations to MST11 on pYK21 with the QuickChange II site-directed mutagenesis kit (Agilent Technologies, Fort Worth, TX, U.S.A.). All the resulting vectors were confirmed by sequencing analysis and were transformed into the mst11 mutant yk86 (Zhao et al. 2005).
Assays for appressorium formation, cytorrhysis, penetration, and plant infection.

Conidia harvested from 10-day-old oatmeal agar cultures were adjusted to a concentration of $1 \times 10^{5}$ conidia/ml in sterile distilled water and were used for appressorium formation on plastic coverslips (Fisher Scientific Co., Pittsburgh, PA, U.S.A.) or GelBond membranes (Cambrex, East Rutherford, NJ, U.S.A.) as described (Tucker et al. 2004; Xue et al. 2002). For assaying its effect on germ-tube branching on the hydrophobic side of GelBond membranes, cAMP was added to conidium suspensions to the final concentration of $5 \mathrm{mM}$ cAMP. Appressorium turgor pressure was estimated with the incipient cytorhysis method using PEG-8000 solutions as described (Howard et al. 1991). Conidia were resuspended in $0.25 \%$ gelatin for plant infection assays. Penetration assays with barley leaves were performed as previously described (Kong et al. 2013; Li et al. 2007). Two-week-old seedlings of rice cultivar Nipponbare were used for spray infection assays. Lesion formation was examined $7 \mathrm{dpi}$. The number of lesions formed on 5-cm leaf tips was determined as described previously (Kershaw et al. 1998).

\section{Yeast two-hybrid assays.}

The HybridZap2.1 yeast two-hybrid system (Agilent Technologies) was used to assay protein-protein interactions. The bait or prey vectors of $M S T 11, R A S 1$, and $R A S 2$ were generated in the previous study (Park et al. 2006; Zhao et al. 2005). Primers RasDAF and RasDAR were used to introduce the G23V mutation of RAS2 on pCX96 with the Quickchange II kit. The resulting bait and prey vectors were cotransformed in pairs into yeast strain YRG-2 with the alkali-cation yeast transformation kit (MP Biomedicals, Irvine, CA, U.S.A.). The $\mathrm{Leu}^{+}$and $\mathrm{Trp}^{+}$ transformants were isolated and assayed for $\beta$-galactosidase activities and growth on SD-His medium (Zhou et al. 2011). Yeast cells grown in liquid SD-Leu-Trp medium were assayed for $\beta$-galactosidase activities with $o$-nitrophenyl- $\beta$-D-galactopyranoside as the substrate.

\section{Western blot analysis.}

Total proteins were isolated from vegetative hyphae and were separated on a $10 \%$ sodium dodecyl sulfate-polyacrylamide electrophoresis gel as described previously (Bruno et al. 2004) and were transferred to nitrocellulose membranes (Amersham, Piscataway, NJ, U.S.A.). The ECL Supersignal System (Pierce, Rockford, IL, U.S.A.) was used for Western blot detection with antibodies. Phosphorylation at the TEY dual phosphorylation site of the Pmk1 and Mps1 MAPKs was detected with the PhophoPlus p44/42 MAPK antibody kit (Cell Signaling Technology, Beverly, MA, U.S.A.), following the manufacturer's instructions. Pmk1 expression was detected with the anti-Pmk1 antiserum generated in a previous study (Bruno et al. 2004). The phosphorylation level of Osm1 MAPK was detected with the PhophoPlus p38 MAPK antibody kit (Cell Signaling Technology).

\section{ACKNOWLEDGMENTS}

We thank L. Dunkle and S. Goodwin for critical reading of this manuscript. We also thank G. Park and X. Zhao for fruitful discussions. This work was supported by a grant from the National Research Initiative of the United States Department of Agriculture National Institute of Food and Agriculture (award number: 2010-65110-20439) and the National Basic Research Program of China (2012CB114002).

\section{LITERATURE CITED}

Andrews, D. L., Egan, J. D., Mayorga, M. E., and Gold, S. E. 2000. The Ustilago maydis $u b c 4$ and $u b c 5$ genes encode members of a MAP kinase cascade required for filamentous growth. Mol. Plant-Microbe Interact. 13: 781-786. 
Bauer, P. M., Fulton, D., Boo, Y. C., Sorescu, G. P., Kemp, B. E., Jo, H., and Sessa, W. C. 2003. Compensatory phosphorylation and protein-protein interactions revealed by loss of function and gain of function mutants of multiple serine phosphorylation sites in endothelial nitric-oxide synthase. J. Biol. Chem. 278:14841-14849.

Bruno, K. S., Tenjo, F., Li, L., Hamer, J. E., and Xu, J. R. 2004. Cellular localization and role of kinase activity of $P M K 1$ in Magnaporthe grisea. Eukaryot. Cell 3:1525-1532.

Chao, C. C. T., and Ellingboe, A. H. 1991. Selection for mating competence in Magnaporthe grisea pathogenic to rice. Can. J. Bot. 69:2130-2134.

Choi, W., and Dean, R. A. 1997. The adenylate cyclase gene MAC1 of Magnaporthe grisea controls appressorium formation and other aspects of growth and development. Plant Cell 9:1973-1983.

Dean, R. A., Talbot, N. J., Ebbole, D. J., Farman, M. L., Mitchell, T. K., Orbach, M. J., Thon, M., Kulkarni, R., Xu, J. R., Pan, H., Read, N. D., Lee, Y. H., Carbone, I., Brown, D., Oh, Y. Y., Donofrio, N., Jeong, J. S., Soanes, D. M., Djonovic, S., Kolomiets, E., Rehmeyer, C., Li, W., Harding, M., Kim, S., Lebrun, M. H., Bohnert, H., Coughlan, S., Butler, J., Calvo, S., Ma, L. J., Nicol, R., Purcell, S., Nusbaum, C., Galagan, J. E., and Birren, B. W. 2005. The genome sequence of the rice blast fungus Magnaporthe grisea. Nature 434:980-986.

Ding, S. L., Liu, W., Iliuk, A., Ribot, C., Vallet, J., Tao, A., Wang, Y., Lebrun, M. H., and Xu, J. R. 2010. The Tig1 histone deacetylase complex regulates infectious growth in the rice blast fungus Magnaporthe oryzae. Plant Cell 22:2495-2508.

Dixon, K. P., Xu, J. R., Smirnoff, N., and Talbot, N. J. 1999. Independent signaling pathways regulate cellular turgor during hyperosmotic stress and appressorium-mediated plant infection by Magnaporthe grisea. Plant Cell 11:2045-2058.

Ebbole, D. J. 2007. Magnaporthe as a model for understanding hostpathogen interactions. Annu. Rev. Phytopathol. 45:437-456.

Franck, W. L., Gokce, E., Oh, Y., Muddiman, D. C., and Dean, R. A. 2013. Temporal analysis of the Magnaporthe oryzae proteome during conidial germination and cyclic AMP (cAMP)-mediated appressorium formation. Mol. Cell. Proteomics 12:2249-2265.

Gronwald, W., Huber, F., Grünewald, P., Spörner, M., Wohlgemuth, S., Herrmann, C., and Kalbitzer, H. R. 2001. Solution structure of the Ras binding domain of the protein kinase Byr2 from Schizosaccharomyces pombe. Structure 9:1029-1041.

Gustchina, A., Li, M., Phylip, L. H., Lees, W. E., Kay, J., and Wlodawer, A. 2002. An unusual orientation for Tyr75 in the active site of the aspartic proteinase from Saccharomyces cerevisiae. Biochem. Biophys. Res. Commun. 295:1020-1026.

Howard, R. J., Ferrari, M. A., Roach, D. H., and Money, N. P. 1991 Penetration of hard substrates by a fungus employing enormous turgor pressures. Proc. Natl. Acad. Sci. U.S.A. 88:11281-11284.

Jenczmionka, N. J., Maier, F. J., Lösch, A. P., and Schäfer, W. 2003. Mating, conidiation and pathogenicity of Fusarium graminearum, the main causal agent of the head-blight disease of wheat, are regulated by the MAP kinase gpmk1. Curr. Genet. 43:87-95.

Kankanala, P., Czymmek, K., and Valent, B. 2007. Roles for rice membrane dynamics and plasmodesmata during biotrophic invasion by the blast fungus. Plant Cell 19:706-724.

Kershaw, M. J., and Talbot, N. J. 2009. Genome-wide functional analysis reveals that infection-associated fungal autophagy is necessary for rice blast disease. Proc. Natl. Acad. Sci. U.S.A. 106:15967-15972.

Kershaw, M. J., Wakley, G., and Talbot, N. J. 1998. Complementation of the mpg1 mutant phenotype in Magnaporthe grisea reveals functional relationships between fungal hydrophobins. EMBO J. 17:3838-3849.

Kong, L.-A., Li, G.-T., Liu, Y., Liu, M.-G., Zhang, S.-J., Yang, J., Zhou, X.-Y., Peng, Y.-L., and Xu, J.-R. 2013. Differences between appressoria formed by germ tubes and appressorium-like structures developed by hyphal tips in Magnaporthe oryzae. Fungal Genet. Biol. 56:33-41.

Lee, Y. H., and Dean, R. A. 1993. cAMP regulates infection structure formation in the plant pathogenic fungus Magnaporthe grisea. Plant Cell 5:693-700.

Li, G., Zhou, X., and Xu, J. R. 2012. Genetic control of infection-related development in Magnaporthe oryzae. Curr. Opin. Microbiol. 15:678-684.

Li, L., Ding, S. L., Sharon, A., Orbach, M., and Xu, J. R. 2007. Mirl is highly upregulated and localized to nuclei during infectious hyphal growth in the rice blast fungus. Mol. Plant-Microbe Interact. 20:448-458.

Li, L., Xue, C., Bruno, K., Nishimura, M., and Xu, J. R. 2004. Two PAK kinase genes, CHM1 and MST20, have distinct functions in Magnaporthe grisea. Mol. Plant-Microbe Interact. 17:547-556.

Liu, W., Zhou, X., Li, G., Li, L., Kong, L., Wang, C., Zhang, H., and Xu, J. R. 2011. Multiple plant surface signals are sensed by different mechanisms in the rice blast fungus for appressorium formation. PLoS Pathog. 7:e1001261.

Mey, G., Oeser, B., Lebrun, M. H., and Tudzynski, P. 2002. The biotrophic, non-appressorium-forming grass pathogen Claviceps purpurea needs a Fus3/Pmk1 homologous mitogen-activated protein kinase for colonization of rye ovarian tissue. Mol. Plant-Microbe Interact.15:303-312.

Mitchell, T. K., and Dean, R. A. 1995. The cAMP-dependent protein kinase catalytic subunit is required for appressorium formation and pathogenesis by the rice blast pathogen Magnaporthe grisea. Plant Cell 7:1869-1878.

Müller, P., Weinzierl, G., Brachmann, A., Feldbrügge, M., and Kahmann, R. 2003. Mating and pathogenic development of the Smut fungus Ustilago maydis are regulated by one mitogen-activated protein kinase cascade. Eukaryot. Cell 2:1187-1199.

Park, G., Xue, C., Zhao, X., Kim, Y., Orbach, M., and Xu, J. R. 2006. Multiple upstream signals converge on the adaptor protein Mst50 in Magnaporthe grisea. Plant Cell 18:2822-2835.

Peng, Y. H., Shiao, H. Y., Tu, C. H., Liu, P. M., Hsu, J. T. A., Amancha, P. K., Wu, J. S., Coumar, M. S., Chen, C. H., Wang, S. Y., Lin, W. H., Sun, H. Y., Chao, Y. S., Lyu, P. C., Hsieh, H. P., and Wu, S. Y. 2013 Protein kinase inhibitor design by targeting the Asp-Phe-Gly (DFG) motif: The role of the DFG motif in the design of epidermal growth factor receptor inhibitors. J. Med. Chem. 56:3889-3903.

Ramachander, R., Kim, C. A., Phillips, M. L., Mackereth, C. D., Thanos, C. D., McIntosh, L. P., and Bowie, J. U. 2002. Oligomerizationdependent association of the SAM domains from Schizosaccharomyces pombe Byr2 and Ste4. J. Biol. Chem. 277:39585-39593.

Rispail, N., Soanes, D. M., Ant, C., Czajkowski, R., Grünler, A., Huguet, R., Perez-Nadales, E., Poli, A., Sartorel, E., Valiante, V., Yang, M., Beffa, R., Brakhage, A. A., Gow, N. A. R., Kahmann, R., Lebrun, M.-H., Lenasi, H., Perez-Martin, J., Talbot, N. J., Wendland, J., and Di Pietro, A. 2009. Comparative genomics of MAP kinase and calcium-calcineurin signalling components in plant and human pathogenic fungi. Fungal Genet. Biol. 46:287-298.

Shewchuk, L. M., Hassell, A. M., Ellis, B., Holmes, W. D., Davis, R., Horne, E. L., Kadwell, S. H., McKee, D. D., and Moore, J. T. 2000. Structure of the Tie2 RTK domain: Self-inhibition by the nucleotide binding loop, activation loop, and C-terminal tail. Structure 8:1105-1113.

Smith, D. G., Garcia-Pedrajas, M. D., Hong, W., Yu, Z., Gold, S. E., and Perlin, M. H. 2004. An ste20 homologue in Ustilago maydis plays a role in mating and pathogenicity. Eukaryot. Cell 3:180-189.

Solomon, P. S., Waters, O. D. C., Simmonds, J., Cooper, R. M., and Oliver, R. P. 2005. The Mak2 MAP kinase signal transduction pathway is required for pathogenicity in Stagonospora nodorum. Curr. Genet. 48:60-68.

Steichen, J. M., Kuchinskas, M., Keshwani, M. M., Yang, J., Adams, J. A., and Taylor, S. S. 2012. Structural basis for the regulation of protein kinase A by activation loop phosphorylation. J. Biol. Chem. 287:14672-14680.

Stevenson, B. J., Rhodes, N., Errede, B., and Sprague, G. F., Jr. 1992 Constitutive mutants of the protein kinase STE11 activate the yeast pheromone response pathway in the absence of the $G$ protein. Genes Dev. 6:1293-1304.

Thines, E., Weber, R. W. S., and Talbot, N. J. 2000. MAP kinase and protein kinase A-dependent mobilization of triacylglycerol and glycogen during appressorium turgor generation by Magnaporthe grisea. Plant Cell 12: 1703-1718.

Treiber, D. K., and Shah, N. P. 2013. Ins and outs of kinase DFG motifs. Chem. Biol. 20:745-746.

Tucker, S. L., Thornton, C. R., Tasker, K., Jacob, C., Giles, G., Egan, M., and Talbot, N. J. 2004. A fungal metallothionein is required for pathogenicity of Magnaporthe grisea. Plant Cell 16:1575-1588.

Valent, B., and Khang, C. H. 2010. Recent advances in rice blast effector research. Curr. Opin. Plant Biol. 13:434-441.

van Drogen, F., O’Rourke, S. M., Stucke, V. M., Jaquenoud, M., Neiman, A. M., and Peter, M. 2000. Phosphorylation of the MEKK Ste11p by the PAK-like kinase Ste20p is required for MAP kinase signaling in vivo. Curr. Biol. 10:630-639.

Veneault-Fourrey, C., Barooah, M., Egan, M., Wakley, G., and Talbot, N. J. 2006. Autophagic fungal cell death is necessary for infection by the rice blast fungus. Science 312:580-583.

Wang, C., Zhang, S., Hou, R., Zhao, Z., Zheng, Q., Xu, Q., Zheng, D., Wang, G., Liu, H., Gao, X., Ma, J. W., Kistler, H. C., Kang, Z., and Xu, J. R. 2011. Functional analysis of the kinome of the wheat scab fungus Fusarium graminearum. PLoS Pathog. 7:e1002460.

Wilson, R. A., and Talbot, N. J. 2009. Under pressure: Investigating the biology of plant infection by Magnaporthe oryzae. Nat. Rev. Microbiol. 7:185-195.

Xu, J. R., and Hamer, J. E. 1996. MAP kinase and cAMP signaling regulate infection structure formation and pathogenic growth in the rice blast fungus Magnaporthe grisea. Genes Dev. 10:2696-2706.

Xu, J. R., Staiger, C. J., and Hamer, J. E. 1998. Inactivation of the mitogenactivated protein kinase $M p s 1$ from the rice blast fungus prevents penetration of host cells but allows activation of plant defense responses. Proc. Natl. Acad. Sci. U.S.A. 95:12713-12718. 
Xu, J. R., Urban, M., Sweigard, J. A., and Hamer, J. E. 1997. The CPKA gene of Magnaporthe grisea is essential for appressorial penetration. Mol. Plant-Microbe Interact. 10:187-194.

Xue, C., Park, G., Choi, W., Zheng, L., Dean, R. A., and Xu, J. R. 2002. Two novel fungal virulence genes specifically expressed in appressoria of the rice blast fungus. Plant Cell 14:2107-2119.

Yi, M., and Valent, B. 2013. Communication between filamentous pathogens and plants at the biotrophic interface. Annu. Rev. Phytopathol. 51: 587-611.

Zhang, S., and Xu, J. R. 2014. Effectors and effector delivery in Magnaporthe oryzae. PLoS Pathog. 10:e1003826.

Zhao, X., Kim, Y., Park, G., and Xu, J. R. 2005. A mitogen-activated protein kinase cascade regulating infection-related morphogenesis in Magnaporthe grisea. Plant Cell 17:1317-1329.
Zhao, X., and Xu, J. R. 2007. A highly conserved MAPK-docking site in Mst7 is essential for Pmk1 activation in Magnaporthe grisea. Mol. Microbiol. 63:881-894.

Zheng, L., Campbell, M., Murphy, J., Lam, S., and Xu, J. R. 2000. The $B M P 1$ gene is essential for pathogenicity in the gray mold fungus Botrytis cinerea. Mol. Plant-Microbe Interact. 13:724-732.

Zhou, X., Liu, W., Wang, C., Xu, Q., Wang, Y., Ding, S., and Xu, J. R. 2011. A MADS-box transcription factor MoMcm1 is required for male fertility, microconidium production and virulence in Magnaporthe oryzae. Mol. Microbiol. 80:33-53.

Zhou, X., Zhao, X., Xue, C., Dai, Y., and Xu, J. R. 2014. Bypassing both surface attachment and surface recognition requirements for appressorium formation by overactive ras signaling in Magnaporthe oryzae. Mol. Plant-Microbe Interact. 27:996-1004. 